\title{
Minijobs - Eine riskante Beschäftigungsform beim normativen Übergang zum „Adult-Worker-Model“
}

Die zunehmende Erwerbstätigkeit von Frauen geht vor allem auf das Konto von Teilzeitund Minijobbeschäftigung. Sind Minijobs eine von Frauen erwünschte Erwerbsform im Rahmen stabiler ehelicher Arrangements? Oder bringen sie ein Verarmungsrisiko mit sich und sind damit eine im Lebensverlauf riskante Beschäftigungsform? Gerade über die Bedeutung von Minijobs im Erwerbsverlauf von Frauen ist wenig bekannt. Der Beitrag wendet sich diesen Fragen zu und zeigt zum einen, dass Minijobs in den Erwerbsbiografien von Frauen eine unterschiedliche Rolle spielen. Zum zweiten wird versucht, die sozialen Kontexte auszuleuchten, die für die unterschiedlichen Funktionen und Auswirkungen von Minijobs in weiblichen Erwerbsbiografien relevant sind.

\section{Einleitung}

Die Erwerbstätigkeit von Frauen ist in Deutschland in den letzten Jahren stark angestiegen. Gründe dafür sind sowohl die gewandelten Rollenvorstellungen der zunehmend besser ausgebildeten Frauen, die eine klare berufliche Orientierung haben (Allmendinger 2009) als auch ökonomische Notwendigkeiten. Immer mehr Frauen sorgen als Singles für sich selbst. Doch auch Frauen in Paarhaushalten können sich heute weniger als früher darauf verlassen, lebenslang von einem männlichen Partner finanziell abgesichert zu werden, sei es, weil auch die Erwerbsverläufe von Männern tendenziell instabiler werden, aber auch wegen des gestiegenen Trennungsrisikos und veränderten Unterhaltsrechts nach einer Scheidung (Klenner/Klammer 2009; Sachverständigenkommission 2011). Vor diesem Hintergrund haben sich bei beiden Geschlechtern zunehmend modernere Geschlechterrollenvorstellungen herausgebildet. ${ }^{0}$

Gleiche wirtschaftliche Unabhängigkeit von Frauen und Männern auf der Basis eines eigenen Einkommens ist auch das erste Ziel, das in der Strategie der Europäischen Union für die Gleichstellung von Frauen und Männern (2010-2015) formuliert wird (EU 2011). Dieses Ziel ist Ausdruck des auf europäischer Ebene inzwischen etablierten Leitbildes des Adult-Worker-Model (ErwerbstätigenModell, vgl. Lewis 2004), nach dem grundsätzlich jede und jeder Erwachsene - ob Frau oder Mann - in allen Lebens- phasen den eigenen Lebensunterhalt durch Erwerbstätigkeit selbst bestreiten soll. Dieses neue Leitbild trägt gewandelten Geschlechterrollenvorstellungen ebenso Rechnung wie ökonomischen Notwendigkeiten unter Bedingungen des demografischen Wandels. Das Adult-Worker-Model ist mit der traditionellen geschlechtsspezifischen Spezialisierung innerhalb einer Ehe, der zufolge der Einkommenserwerb zum Unterhalt der Familie die Aufgabe des Mannes, dagegen Hausarbeit, Kinderbetreuung und Pflege die Aufgabe der Frau sei, kaum vereinbar. Denn die Spezialisierung und Rollenteilung geht häufig damit einher, dass Frauen nur einen Zuverdienst erwerben und nicht eigenständig ihren Lebensunterhalt erwirtschaften. Und genau daran hat sich in Deutschland trotz der wachsenden Erwerbstätigkeit von Frauen bislang nur wenig geändert, da hier der größte Teil des Zuwachses der Frauenerwerbstätigkeit auf das Konto von Teilzeit-, und darunter von Minijobbeschäftigung geht (Sachverständigenkommission 2011).

\footnotetext{
( Das traditionelle männliche Familienernährermodell wird nur noch von $40 \%$ aller Westdeutschen und sogar von nur $17 \%$ der Ostdeutschen unterstützt, wobei diese Werte unter den Jüngeren noch deutlich niedriger waren (Statistisches Bundesamt 2006). Erfragt wurde die Zustimmung zur Aussage „Es ist für alle Beteiligten besser, wenn der Mann voll im Berufsleben steht und die Frau zuhause bleibt und sich um den Haushalt und die Kinder kümmert".
} 
Minijobs als Hauptbeschäftigung werden überwiegend von Frauen ausgeübt. ${ }^{2}$ Ca. 3,3 Mio. Frauen haben ausschließlich einen Minijob (sowie 1,6 Mio. Männer: vgl. HBSDatenbank 2011). Gegenwärtig wird davon ausgegangen, dass die geringen Arbeitseinkommen aus Minijobs „mit anderen Einkommensarten kombiniert [werden], mit einem Hauptverdienst, mit Partnereinkommen oder Sozialtransfers" und daher ,aus Sicht der Beschäftigten eine Ergänzung anderer Einkünfte darstellen“ (Eichhorst et al. 2010, S. 25). Diese Beschäftigungsverhältnisse sind daher zwar innerhalb des konservativen wohlfahrtsstaatlichen Leitbilds der Versorgerehe, in der verheiratete Frauen keine eigenständige, sondern eine abgeleitete soziale Sicherung über den Ehemann haben, ein konsistentes Element. Doch dem neuen Leitbild des Adult-Worker-Model widersprechen Minijobs, weil sie weder aktuell noch zukünftig beim Altersrentenbezug eine existenzsichernde Funktion erfüllen können.

Insbesondere für verheiratete Mütter erscheinen Minijobs vor dem Hintergrund der wohlfahrtstaatlichen Regelungen sowie der Bedingungen der Vereinbarkeit von Familie und Beruf in einer kurzfristigen Perspektive auf den ersten Blick attraktiv oder zumindest als annehmbar. Zum einen ist die Kinderbetreuung für Kinder unter drei Jahren wie auch für jüngere Schulkinder in Deutschland mit Blick auf eine volle Erwerbstätigkeit der Eltern unzureichend ausgebaut (Statistisches Bundesamt 2011; Kassner 2011). Und selbst wenn ein Kind einen Kindergartenplatz hat, erlauben die Öffnungszeiten der Kinderbetreuungseinrichtungen häufig nur eine kurze Teilzeitbeschäftigung. Zum anderen setzt der Staat nach wie vor Anreize, die das traditionelle Geschlechtermodell mit männlichem Familienernährer und weiblicher Hinzuverdienerin fördern. „Die Abgabenprivilegierung geringfügiger Beschäftigung im Sozial- und Steuerrecht begründet im Zusammenwirken mit der Institution der Ehe das Rollenmodell des verheirateten Familienernährers und der geringfügig hinzuverdienenden Ehefrau (...).“ (Sachverständigenkommission 2011, S. 55). Doch aus der Perspektive der Geschlechtergleichstellung in der Lebensverlaufsperspektive erweisen sich Minijobs häufig als Sackgasse. Daher spricht sich die Sachverständigenkommission für den Ersten Gleichstellungsbericht „mit Nachdruck für die Abschaffung der Subventionierung von geringfügigen Beschäftigungsverhältnissen aus“" (ebd., S. 135).

Auch wenn auf der Hand liegt, dass Minijobs nicht den Lebensunterhalt decken können, ist bisher wenig empirisch darüber bekannt, welche Rolle sie im Lebensverlauf der in Minijobs Beschäftigten tatsächlich spielen. Die meisten Forschungsergebnisse basieren auf Querschnittsanalysen und berücksichtigen nicht die Längsschnittdimension. Die wenigen vorliegenden Längsschnittanalysen zeigen, dass ein hoher Anteil westdeutscher Frauen mit Kindern in ihren Erwerbsverläufen für mindestens ein Jahr durchgängig teilzeiterwerbstätig war (Klammer/Tillmann 2001). Im Durchschnitt waren westdeutsche Frauen im Zeitraum 1995 bis 2003 zwei Jahre teilzeiterwerbstätig (Schäfer/Vogel 2005). Darüber hinaus ist bekannt, dass Erwerbsverläufe sich in den letzten Jahren stärker ausdifferenziert haben (Schmidt/ Schmitt 2005), und dass es auch unter Frauen sehr unterschiedliche Erwerbsverläufe gibt (vgl. Hacket 2011). Weiterhin wissen wir, dass Minijobs kaum eine „Brückenfunktion“ erfüllen, denn nur $9 \%$ der Minijobberinnen und Minijobber wechseln aus dem Minijob in ein Normalarbeitsverhältnis (Tschersich et al. 2010).

Eine wesentliche Wissenslücke besteht hinsichtlich der Frage, welche Auswirkungen Minijobphasen auf die weiteren Erwerbschancen haben. Für Teilzeitarbeit insgesamt sind im Längsschnitt Einkommenseinbußen und „Vernarbungseffekte" nachgewiesen (vgl. Klammer et al. 2008; Fouarge/Muffels 2009). Dies wird auch durch die Querschnittsanalysen gestützt; hier zeigen sich deutliche Einkommenseinbußen im Stundenlohn bei Arbeitsverhältnissen im Bereich von zehn bis 15 Stunden pro Woche (Wolf 2010). Auch Altersarmut wird für Beschäftigte in Minijobs anhand von Makrodaten konstatiert (Steffen 2010), jedoch auf Individualebene bisher nicht belegt.

Im Mittelpunkt des folgenden Beitrags stehen folgende Fragen: Welche Rolle spielen Minijobs im Erwerbsverlauf von Frauen? Über welche Zeiträume werden Minijobs ausgeübt? Sind Minijobs von Frauen tatsächlich, wie im Allgemeinen angenommen, eine Ergänzung zu einem ausreichenden Partnereinkommen, das eine Absicherung der Familie erlaubt? Werden Minijobs vor diesem Hintergrund dauerhaft freiwillig ausgeübt? Können auch Erwerbsverläufe, die zeitweilig Minijobs einschließen, zu einer eigenständigen Sicherung des Lebensunterhalts gemäß dem AdultWorker-Model führen?

Um Antworten auf diese Fragen zu finden, untersuchen wir im Längsschnitt, in welchen Typen von Erwerbsverläufen Minijobs vorkommen und bei welchen Frauen sie sich konzentrieren. Wir werden erstens zeigen, dass Minijobs nicht allein von Frauen in Partnerschaften im Rahmen stabiler Teilzeitverläufe ausgeübt werden, sondern auch als prekärer Bestandteil innerhalb diskontinuierlicher Erwerbsverläufe eine Rolle spielen. Wir zeigen zweitens, dass Minijobs vor allem in stabilen Erwerbsverläufen überwiegend freiwillig ausgeübt werden. Schließlich stellen wir drittens dar, dass auch für diejenigen, die unter den gegebenen Anreizstrukturen mit dem Minijob zufrieden sind und bei denen aktuell kein Armutsrisiko besteht, Minijobs in der Lebensverlaufsperspektive riskant sind.

Nach Darstellung von Daten und Methoden der diesen Ausführungen zugrunde liegenden Analyse (Abschnitt 2) gehen wir zunächst auf jene Erwerbsverlaufstypen ein, in denen Teilzeiterwerbstätigkeit und Minijobs eine Rolle spielen (3). Danach werden soziökonomische Merkmale von Frauen mit Minijobs, wie Haushaltszusammenhang,

(2) Daneben werden Minijobs als Hauptbeschäftigung vor allem von Rentnern, Schülern und Studierenden, also außerhalb der Haupterwerbsphase, ausgeübt (vgl. Bäcker/ Neuffert in diesem Heft). 
Einkommenssituation und Freiwilligkeit der Beschäftigungsform, analysiert (4). Anschließend ziehen wir ein Fazit (5).

\section{Daten und Methoden}

In diesem Beitrag betrachten wir Erwerbsverläufe von Frauen der Geburtskohorten 1965 bis 1979 für den Beobachtungszeitraum von 2001 bis 2007. Basis ist das Soziooekonomische Panel (SOEP). ${ }^{\boldsymbol{\beta}}$ Dabei wird berücksichtigt, wie lange Kinder im Haushalt lebten, da sich die Erwerbsverläufe von Frauen mit Kindern und ohne Kinder stark unterscheiden. Daher differenzieren wir von Anfang an zwischen Verläufen, in denen Frauen mehr als $60 \%$ der beobachteten Zeit mit Kindern im Haushalt leben, und solchen, in denen Frauen mehr als $60 \%$ der Zeit ohne Kinder im Haushalt leben. Damit werden in den „Verläufen ohne Kind(er)“ auch Mütter erfasst, deren Kinder erst am Ende des Beobachtungszeitraumes geboren worden sind, wie auch Mütter, deren Kinder im Beobachtungszeitraum nur weniger als drei Jahre im Kindesalter (hier definiert bis 16 Jahre) waren und dann aus dem Kindesalter „herausgewachsen“ sind. Eine solche Abgrenzung war aber notwendig, um hinreichende Fallzahlen für die weitere Analyse mit tieferen Differenzierungen zu erhalten. ${ }^{\circledR}$ Im Folgenden wird verkürzt von „Frauen mit Kindern“ bzw. von „Frauen ohne Kinder“ gesprochen. Zudem beziehen sich die Aussagen immer - soweit nicht anders angegeben - auf den bereits genannten Analysezeitraum der Erwerbsverläufe (2001 bis 2007) und hier auf Frauen der o. g. Geburtskohorten. Diese Frauen waren im untersuchten Zeitraum zwischen 34 bis 43 Jahre (älteste Gruppe) und 23 bis 30 Jahre (jüngste Gruppe) alt. Wegen der besseren Lesbarkeit des Textes wird darauf nicht immer hingewiesen.

Zur Konstruktion der Erwerbsverläufe wurden aus den Erwerbsstatusinformationen ${ }^{\circledR}$ jeden Monats zwischen 2001 und 2007 zeitlich geordnete Sequenzen gebildet und anschließend mit der sogenannten Sequenzanalyse ${ }^{\bullet}$ analysiert: Mit dieser Methode ist es beispielsweise möglich zu ermitteln, wie oft und wie lange in der beobachteten Zeit
Teilzeiterwerbstätigkeit oder Vollzeiterwerbstätigkeit vorkam. Zudem ist festzustellen, in welcher Reihenfolge die verschiedenen Erwerbsstatus in der Beobachtungszeit auftraten. Damit sind Sequenzen als eine zeitlich geordnete Liste von aufeinander folgenden Elementen des Erwerbsverlaufs zu verstehen. Gleiche Elemente (also gleicher Erwerbsstatus) in aufeinanderfolgenden Monaten werden zu sogenannten Episoden zusammengefasst. Die Sequenzen sowie die darin enthaltenen Episoden wurden mit der Sequenzanalyse zunächst deskriptiv analysiert. Anschließend wurden alle Sequenzen mit dem Optimal-Matching-Verfahren miteinander verglichen und die daraus resultierenden numerischen Unterschiede (Distanzen) zwischen den Sequenzen wurden mit einer Clusteranalyse ${ }^{\boldsymbol{0}} \mathrm{zu}$ verschiedenen Typen von Erwerbsverläufen gruppiert.

\section{Erwerbsverlaufstypen}

Mit dem beschriebenen Verfahren lassen sich die beobachteten Erwerbsverläufe der Frauen mit Kindern und der Frauen ohne Kinder über die Jahre 2001 bis 2007 in verschiedene Erwerbsverlaufstypen gruppieren. Für Frauen mit Kindern finden sich vier verschiedene Erwerbsverlaufstypen; für Frauen ohne Kinder ergeben sich drei Erwerbsverlaufstypen. Die Verlaufstypen unterscheiden sich stark nach den Abfolgen der Erwerbsstatus in der beobachteten Zeit sowie nach den kumulierten Zeitanteilen der einzelnen Erwerbsstatus an der beobachteten Zeit. Die Verteilung aller Typen und grundlegende Kennzeichen werden in Übersicht 1 zusammengefasst (vgl. ausführlich Klenner/Schmidt 2011).

Frauen leben demnach nicht nur einen oder zwei verschiedene Erwerbsverläufe, sondern Verläufe unterschiedlichster Art: Bei Frauen mit Kindern und Frauen ohne Kinder kommen jeweils ungefähr zur Hälfte stabile Teilzeit- und Vollzeiterwerbsverläufe ebenso vor wie zur anderen Hälfte diskontinuierliche und prekäre Verläufe. Dabei sind es keineswegs nur Mütter, die in Teilzeiterwerbstätigkeit und Minijobs arbeiten, sondern auch Frauen ohne Kinder.
3 Dabei handelt es sich um SOEP v25. Zur Datenbasis siehe www.diw.de/soep.

4 Denn über $70 \%$ der Frauen der Stichprobe (hochgerechnet) lebten irgendwann zwischen 2001 und 2007 mit Kindern im Haushalt, das heißt die Gruppe der Frauen ganz ohne Kind(er) während der gesamten Zeit ist sehr klein. Bei der Überprüfung anderer zeitlicher Abgrenzungen, wie z. B. 75 oder $100 \%$ der Beobachtungszeit mit bzw. ohne Kind, zeigten sich jedoch vergleichbare Unterschiede und Trends zu der endgültig gewählten $60 \%$-Abgrenzung.

(5 Das ist die Angabe des jeweiligen Erwerbsstatus: Teilzeit/ geringfügig/Minijob, Vollzeiterwerbstätigkeit inkl. Kurzarbeit, Arbeitslosigkeit, Ausbildung/Schule, Erziehungszeit, Haushaltstätigkeit, andere Status.
(6 Die Methode der Sequenzanalyse wird zunehmend in den Sozialwissenschaften angewendet (Brüderl/Scherer 2004; Brzinsky-Fay et al. 2006). Sie ermöglicht deskriptive Einblicke in den Aufbau und die Struktur gesamter Sequenzen von festgelegten "Zuständen“.

(7) Dabei werden unter Berücksichtigung der Lage und der jeweiligen Dauer des entsprechenden Status alle Sequenzen miteinander verglichen (vgl. Aisenbrey/Fasang 2007). Mit einem mehrstufigen Clusterverfahren werden die daraus gewonnenen Distanzmaße zwischen den Sequenzen zu in sich möglichst homogenen und untereinander möglichst heterogenen Gruppen zusammengefasst. 


\section{Charakteristika der Erwerbsverlaufstypen von Frauen in Deutschland ${ }^{1}$}

\section{Erwerbsverlaufstypen von Frauen mit Kindern}

\begin{tabular}{|c|c|c|}
\hline Verlaufstyp & $\begin{array}{l}\text { Anteil an allen } \\
\text { Frauen mit Kindern }\end{array}$ & Wichtigste Kennzeichen des Typs \\
\hline „Teilzeit“ (MK-1) & $39,6 \%(N=406)$ & $\begin{array}{l}29 \% \text { der Frauen dieses Typs sind „ununterbrochen teilzeiterwerbstätig“ ohne andere } \\
\text { Tätigkeiten. Dieser Anteil wird gestützt durch die, kumuliert, fast durchgängige Zeit in } \\
\text { Teilzeiterwerbstätigkeit. } 64 \% \text { der Frauen dieses Typs haben Minijobepisoden. }\end{array}$ \\
\hline $\begin{array}{l}\text { "Diskontinuierlich- } \\
\text { prekär" (MK-2) }\end{array}$ & $27,5 \%(N=255)$ & $\begin{array}{l}\text { DieserTyp ist durch sehr viele verschiedene Abfolgen verschiedener Status gekennzeichnet. } \\
\text { Dabei sind sie kumuliert zu etwa jeweils einem Viertel der Zeit in Teilzeit und Elternzeit und } \\
\text { etwas kürzer in Vollzeit und im Haushalt tätig bzw. arbeitslos. } 41 \% \text { der Frauen dieses Verlaufs } \\
\text { weisen mindestens eine Minijobtätigkeit auf. }\end{array}$ \\
\hline „Hausarbeit" (MK-3) & $13,6 \%(\mathrm{~N}=136)$ & $\begin{array}{l}\text { Fast } 50 \% \text { der Frauen dieses Typs sind entweder durchgehend im Haushalt oder zuerst } \\
\text { in Erziehungszeit und dann im Haushalt tätig. Frauen dieses Typs verbringen kumuliert drei } \\
\text { Viertel der Beobachtungszeit im Haushalt. }\end{array}$ \\
\hline „Stabile Vollzeit“ (MK-4) & $19,3 \%(\mathrm{~N}=197)$ & $\begin{array}{l}36 \% \text { der Frauen dieses Typs sind durchgehend vollzeiterwerbstätig. Dementsprechend sind } \\
\text { die Frauen dieses Typs über } 80 \% \text { der Beobachtungszeit vollzeiterwerbstätig. }\end{array}$ \\
\hline
\end{tabular}

\section{Erwerbsverlaufstypen von Frauen ohne Kinder}

\begin{tabular}{l|l|l}
\hline Verlaufstyp & $\begin{array}{l}\text { Anteil an allen } \\
\text { Frauen ohne Kinder }\end{array}$ & Wichtigste Kennzeichen des Typs \\
\hline & & $\begin{array}{l}19 \% \text { der Frauen dieses Typs haben häufige erwerbszeitlich reduzierende Wechsel von } \\
\text { Teilzeit in Erziehungszeit oder von Vollzeiterwerbstätigkeit in Teilzeiterwerbstätigkeit und } \\
\text { bzw. in Erziehungszeit. Die Frauen dieses Typs sind fast die Hälfte der beobachteten Zeit } \\
\text { vollzeiterwerbstätig, jedoch auch jeweils ein Fünftel der Zeit in Teilzeit und in Elternzeit. } \\
38 \% \text { von innen sind mindestens einmal im Minijob tätig. }\end{array}$ \\
\hline "Diskontinuierlich- & $21,0 \%(\mathrm{~N}=141)$ &
\end{tabular}

\begin{tabular}{|l|l|l|l}
\hline \begin{tabular}{|l} 
"Diskontinuierlich- \\
prekär" (OK-2)
\end{tabular} & $\begin{array}{l}\text { DieserTyp ist durch sehr viele verschiedene Abfolgen verschiedener Status gekennzeichnet. } \\
\text { Insgesamt sind die Frauen dieses Typs knapp die Hälfte der Zeit teilzeiterwerbstätig und kürzere } \\
\text { Zeit in Erziehungszeit und kürzere Zeit arbeitslos. } 34 \% \text { dieses Typs haben mindestens eine } \\
\text { Minijobepisode. }\end{array}$ \\
\hline
\end{tabular}

Minijobs kommen vorwiegend in vier Erwerbsverlaufstypen (MK-1, MK-2, OK-1 und OK-2) vor (vgl. Übersicht 1). Frauen arbeiten in den verschiedenen Verlaufstypen unterschiedlich lange in Minijobs: Im Teilzeitverlauf der Mütter (MK-1) sind sie über ein Viertel der Beobachtungszeit in Minijobs. Im diskontinuierlich-prekären Verlauf der Mütter (MK-2) arbeiten diese im Durchschnitt 10\% der Beobachtungszeit in Minijobs. Im diskontinuierlich-prekären Verlauf der Frauen ohne Kinder (OK-2) arbeiten die Frauen durchschnittlich $11 \%$ der Zeit in Minijobs. Im diskontinuierlich-reduzierenden Verlauf (OK-1) haben zwar 38 \% der Frauen des Typs mindestens einmal einen Minijob, doch sind diese Frauen insgesamt im Durchschnitt nur 7\% der Beobachtungszeit in Minijobs tätig.

Betrachten wir, was auf den Minijob folgt, dann zeigt sich: Minijobs stellten nur für einen kleinen Teil der Frauen aller Verläufe eine Brücke zwischen vorhergehender Nichterwerbstätigkeit und nachfolgender (Vollzeit- oder Teilzeit-) Erwerbstätigkeit dar, die meisten verbleiben im Minijob. Das gilt für Mütter ebenso wie für Frauen ohne Kinder der Verlaufstypen OK-1 und OK-2. Vor allem im Verlaufstyp OK-2 kehrt die Mehrheit der Frauen nach der Minijobtätigkeit zurück in Nichterwerbstätigkeit. Im diskontinuierlich-prekären Verlaufstyp MK-2 kommen noch weniger Frauen aus Nichterwerbstätigkeit über einen Minijob in Erwerbstätigkeit. $^{\mathbf{8}}$

8 Die Auswertungen können auf Anfrage zu Verfügung gestellt werden. 
TABELLE 1

\section{Sozioökonomische Eigenschaften der Frauen in ausgewählten Verlaufstypen}

\begin{tabular}{|c|c|c|c|c|}
\hline \multirow{3}{*}{$\begin{array}{l}\text { Verlaufstyp } \\
\begin{array}{l}\text { Kumulierte anteilige Zeit } \\
\text { mit Partner im Haushalt }\end{array} \\
\text { Geburtsjahrgänge / Alter }\end{array}$} & \multicolumn{4}{|c|}{ Frauen mit Kindern } \\
\hline & \multicolumn{2}{|c|}{$\begin{array}{l}\text { Teilzeit-Verlauf (MK-1) } \\
\qquad 90 \%^{1)}\end{array}$} & \multicolumn{2}{|c|}{$\begin{array}{l}\text { Diskontinuierlich-prekärer Verlauf (MK-2) } \\
\qquad 74 \%\end{array}$} \\
\hline & $\begin{array}{l}\text { Anteil Kohorte an } \\
\text { Verlaufstyp (in \%) }\end{array}$ & $\begin{array}{l}\text { Anteil kumulierte Zeit } \\
\text { im Minijob (in \%) }\end{array}$ & $\begin{array}{l}\text { Anteil Kohorte an } \\
\text { Verlaufstyp (in \%) }\end{array}$ & $\begin{array}{l}\text { Anteil kumulierte Zeit } \\
\text { im Minijob (in \%) }\end{array}$ \\
\hline Alle Geburtskohorten & & 26 & & 10 \\
\hline 1965-67: 34-43 Jahre & $39^{21}$ & $22^{3)}$ & 20 & 11 \\
\hline 1968-70: 31-39 Jahre & 36 & 24 & 24 & 14 \\
\hline 1971-73: 28-36 Jahre & 17 & 32 & 28 & 7 \\
\hline 1974-76: 25-33 Jahre & 7 & 35 & 16 & 12 \\
\hline 1977-79: 23-30 Jahre & - & - & - & - \\
\hline Region & $\begin{array}{l}\text { Anteil Region an } \\
\text { Verlaufstyp (in \%) }\end{array}$ & & $\begin{array}{l}\text { Anteil Region an } \\
\text { Verlaufstyp (in \%) }\end{array}$ & \\
\hline Westdeutschland & 89 & 28 & 76 & 22 \\
\hline Ostdeutschland & 11 & 5 & 24 & 6 \\
\hline Höchster berufl. Abschluss 2007 & $\begin{array}{c}\text { Anteil } \\
\text { beruflicher Abschluss } \\
\text { an Verlaufstyp (in \%) }\end{array}$ & & $\begin{array}{c}\text { Anteil } \\
\text { beruflicher Abschluss } \\
\text { an Verlaufstyp (in \%) }\end{array}$ & \\
\hline $\begin{array}{l}\text { (noch) ohne } \\
\text { beruflichen Abschluss }\end{array}$ & 13 & 40 & 23 & 13 \\
\hline Beruflicher Abschluss & 77 & 25 & 65 & 10 \\
\hline Hochschulabschluss & 10 & 14 & - & - \\
\hline
\end{tabular}

Frauen ohne Kinder

Diskontinuierlich-prekärer Verlauf (OK-2) $63 \%$

\begin{tabular}{|c|c|}
\hline $\begin{array}{c}\text { Anteil Kohorte an } \\
\text { Verlaufstyp (in \%) }\end{array}$ & $\begin{array}{c}\text { Anteil kumulierte Zeit } \\
\text { im Minijob (in \%) }\end{array}$ \\
\hline- & 11 \\
\hline 20 & 13 \\
\hline 20 & 14 \\
\hline 20 & 8 \\
\hline 28 & 13 \\
\hline $\begin{array}{c}\text { Anteil Region an } \\
\text { Verlaufstyp (in \%) }\end{array}$ & 13 \\
\hline 78 & 9 \\
\hline 22 & \\
\hline 20 & \\
\hline $\begin{array}{c}\text { Anteil } \\
\text { an Verlaufstyp (in \%) }\end{array}$ & \\
\hline
\end{tabular}

\begin{tabular}{c|c}
\hline 23 & 16 \\
\hline 49 & 11 \\
\hline 27 & 9 \\
\hline
\end{tabular}

1) Lesehilfe: Frauen dieses Typs leben $90 \%$ der Beobachtungszeit mit einem Partner im Haushalt.

2) Lesehilfe: Den Teilzeiterwerbsverlaufstyp leben zu $39 \%$ Frauen (mit Kindern) der Kohorte 1965-67, die im Beobachtungszeitraum zwischen $34-43$ Jahre alt sind.

3) Lesehilfe: Die Frauen des Teilzeitverlaufstyps der Geburtskohorte 1965-67 arbeiten im Durchschnitt kumuliert $22 \%$ der beobachteten sieben Jahre in einem Minijob.

*) Fehlende Werte auf 100 aufgrund zu niedriger Fallzahlen in den einzelnen Zellen; - Fallzahlen $<15$

Quelle: SOEP 2001-2007, hochgerechnet; Berechnungen der Autorinnen; Prozentangaben gerundet.

\section{Erwerbsverlaufstypen mit Minijobs}

Wir beschränken unsere weiteren Analysen auf die Verläufe, in denen Minijobs im Anteil und in der Dauer eine wichtige Rolle spielen. ${ }^{\circledR}$ Dieses sind die „Teilzeiterwerbsverläufe“ (MK-1), die „diskontinuierlich-prekären Verläufe“ der Frauen mit Kindern (MK-2) sowie der Frauen ohne Kinder (OK-2).

Zur näheren Beschreibung der ausgewählten Verlaufstypen mit bedeutendem Minijobanteil werden nun die Abfolgen der verschiedenen Erwerbsstatus, die Anzahl der Episoden in ausgewählten Erwerbsstatus sowie die kumulierten Zeitanteile in ausgewählten Erwerbsstatus innerhalb der Verlaufstypen als weitere Indikatoren herangezogen. Ein Drittel der Frauen mit Kindern des Teilzeiterwerbsverlaufstyps (MK-1) sind durchgängig teilzeiterwerbstätig (Übersicht 1). Wenn die Frauen dieses Typs mindestens einmal in einem Minijob arbeiteten, dann machte die Minijobtätigkeit eine lange Zeit (durchschnittlich die Hälfte der Beobachtungszeit) aus. Demgegenüber sind in den anderen beiden diskontinuierlichen prekären Verläufen der Frauen mit (MK-2) und ohne Kinder (OK-2) die Anteile der Teilzeiterwerbstätigkeiten und der Minijobtätigkeiten an dem Beobachtungszeitraum kürzer und, da sie in mehreren getrennten Episoden erfolgen, diskontinuierlicher. ${ }^{\circledR}$

\subsection{Eigenschaften der Frauen in ausgewählten Verlaufstypen}

Um zu klären, welche Frauen sich in den einzelnen Verlaufstypen wiederfinden, sind in Tabelle 1 einige soziodemografische Merkmale und entsprechende Verlaufstypenmerkmale der ausgewählten Minijob-Erwerbsverlaufstypen zusammengefasst.

(1) Charakteristisch für den Teilzeiterwerbsverlaufstyp (MK-1) ist, dass es sich hier fast ausschließlich um Frauen

(9) Hierbei war auch eine ausreichende Fallzahl zu berücksichtigen.

(1) Diese Ergebnisse sind nicht in Übersicht 1 dargestellt. Sie werden auf Anfrage zur Verfügung gestellt. 
aus Westdeutschland ${ }^{\mathbb{1}}$ handelt. In diesem Verlaufstyp leben die Frauen fast die gesamte Beobachtungszeit mit einem Partner im Haushalt. Es handelt sich bei diesem Erwerbsverlaufstyp offenbar um Frauen, die im Rahmen einer Versorgerehe auf den Partner bauen.

Im Teilzeiterwerbsverlaufstyp sind die ältesten Frauen unserer Untersuchungsgruppe (geboren zwischen 1965 und 1967) am stärksten vertreten (39\%), jedoch arbeiten sie kürzere Zeiträume als andere Altersgruppen in einem Minijob, nämlich $22 \%$ der Beobachtungszeit. Demgegenüber machen Frauen der jüngeren Kohorte (1974-1976 geboren) nur einen kleinen Anteil (7\%) an diesem Verlaufstyp aus. Diese Frauen arbeiten jedoch zu einem weit überdurchschnittlichen Anteil von über $35 \%$ der Beobachtungszeit in Minijobs. Das lässt folgende Schlussfolgerungen zu: Nur noch wenige von den jüngeren Frauen lassen sich auf diesen Teilzeitverlauf ein, bei dem sie fast vollständig vom Partner abhängig sind, aber diese wenigen haben entweder ihrem Lebenskontext geschuldete Gründe, länger in Minijobs zu verweilen - etwa, weil ihre Kinder noch kleiner sind -, oder diesen Frauen gelingt es schwerer als den Älteren, in regulären Teilzeitverhältnissen zu arbeiten.

Über drei Viertel der Frauen dieses Typs haben eine berufliche Ausbildung. Die relativ wenigen Frauen dieses Typs, die keine Ausbildung haben, sind überdurchschnittlich lange in Minijobs tätig, wechseln also noch weniger als andere in reguläre Teilzeit oder Vollzeit. Die kumulierten Zeiten bisheriger Erwerbstätigkeiten bzw. Arbeitslosigkeitsphasen bis zum Beginn des Beobachtungszeitraums weisen auf Pfadabhängigkeiten im Erwerbsstatus hin: Die Frauen des Teilzeiterwerbsverlaufstyps waren auch vor dem Beobachtungszeitraum überdurchschnittlich lange bereits in Teilzeit erwerbstätig. ${ }^{\text {. }}$

(2) Im diskontinuierlich-prekären Verlauf der Mütter (MK2) lebt ein überproportionaler Anteil der Frauen (24\%) überwiegend in Ostdeutschland. Über zwei Drittel der Frauen dieses Erwerbsverlaufstyps haben einen beruflichen Abschluss. In diesem Verlaufstyp leben die Frauen knapp drei Viertel der Zeit mit einem Partner im Haushalt. Das heißt aber auch, dass die Frauen durchschnittlich ein Viertel der Zeit nicht auf den absichernden Partner bauen können. Das hohe Armutsrisiko Alleinerziehender (Statistisches Bundesamt 2010) kann mit solchen Verläufen im Zusammenhang stehen.

(3) Die Frauen ohne Kinder im diskontinuierlich-prekären Verlauf(OK-2) leben nur zu zwei Drittel der Zeit mit Partner im Haushalt. Hier ist also der Lebensunterhalt noch seltener durch einen verdienenden Partner gesichert. In diesem Typ sind am häufigsten die jüngsten Frauen (19771979 geboren) zu finden, welche auch am längsten in Minijobs arbeiten. Überproportional viele Frauen dieses Verlaufstyps leben in Ostdeutschland. Zwar sind etwas mehr als ein Fünftel der Frauen dieses Typs (noch) ohne beruflichen Abschluss, aber die Hälfte hat einen solchen und knapp ein Drittel hat sogar einen Hochschulabschluss. $10 \%$ dieser Frauen mit Hochschulabschluss waren danach mindestens einmal in einem Minijob tätig. ${ }^{(3)}$

Frauen ohne beruflichen Abschluss sind im Vergleich aller Typen überwiegend in den diskontinuierlich-prekären Verläufen zu finden. Gerade diese am wenigsten qualifizierten Frauen arbeiten in allen Typen jeweils überdurchschnittlich lange in Minijobs. Hierfür könnten die Regelungen im Sozialgesetzbuch Zweites Buch (SGB II) mit verantwortlich sein, denn durch den Vorrang der Arbeitsaufnahme vor einem Leistungsbezug bei gleichzeitig fehlenden versicherungspflichtigen Arbeitsplätzen in diesem Segment sind diese Frauen mit niedriger Qualifikationen unter Umständen dazu gezwungen, Minijobs anzunehmen. In allen drei Verläufen sind jedoch auch relativ viele Frauen mit mittleren Berufsabschlüssen anzutreffen. Diese Gruppe kommt schneller wieder aus einer Minijobbeschäftigung heraus. Gleiches gilt auch für die Frauen mit Hochschulabschluss: Diese Frauen können zwar auch in diskontinuierlichen, prekären Erwerbsverläufen landen, sie bleiben jedoch nur über kürzere Zeiträume hinweg in Minijobs.

Bemerkenswert ist auch, dass in allen drei Typen die jüngsten Frauen überdurchschnittlich lange in Minijobs arbeiten.

Die Längsschnittbetrachtung gibt auch Anhaltspunkte für Abhängigkeiten des weiteren Erwerbs- bzw. Lebensverlaufs von vorherigen Erwerbsstatus. ${ }^{(1)}$ Das Erwerbsverhalten von Frauen im Teilzeitverlauf innerhalb unseres Beobachtungszeitraums baut auf überdurchschnittlich langen Teilzeiterwerbserfahrungen bereits vor 2001 auf (Abbildung 1). Dies ist auch die Gruppe von Frauen, die am längsten in Minijobs arbeitet. Auch die Frauen ohne Kinder im diskontinuierlich-prekären Verlauf haben überdurchschnittlich lange vorher in Teilzeit gearbeitet. ${ }^{\oplus}$ Hatten die Frauen mehr oder längere Vorerfahrungen mit Arbeitslosigkeit (Abbildung 2), dann finden sie sich später eher in den beiden diskontinuierlich-prekären Verläufen wieder.

\section{(1) Diese Frauen leben mehr als vier Jahre der Beobachtungszeit in Westdeutschland.}

(12) Diese Unterschiede sind auf dem $5 \%$-Niveau signifikant und hier nicht gesondert dargestellt.

(13 Dabei handelt es sich um die ausschließliche Tätigkeit in einem Minijob und nicht um einen Minijob in Nebentätigkeit. Dabei ist der Anteil derer, die nach Abschluss des Studium direkt in einen Minijob münden, sehr gering (vier Frauen des Verlaufstyps OK-2). Diese geringen Fallzahlen sind, wie bereits erwähnt, auch der vorliegenden Stichprobenabgrenzung und dem begrenzten Beobachtungszeitraum geschuldet.

(44) Generell sind Lebensverläufe durch ihre Selbstreferenzialität interdependent (vgl. z. B. Blossfeld/Huinink 2002)

(5) Die Unterschiede zwischen den Verlaufstypen sind signifikant. 
ABB.

\section{Pfadabhängigkeit von Erwerbsverläufen - Vorerfahrung in Teilzeitarbeit -}

durchschnittliche Anzahl Jahre vor 2001

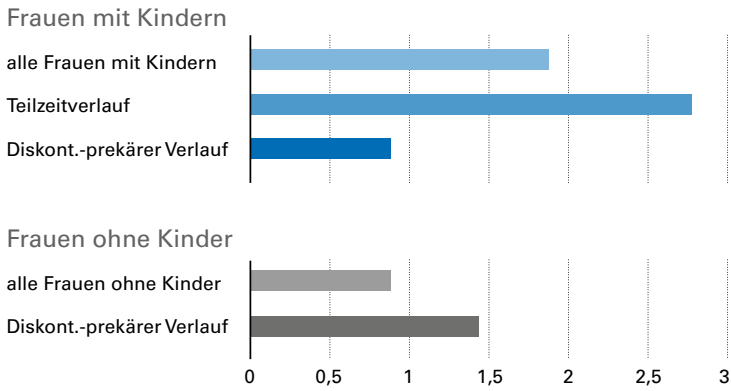

Quelle. SOEP, verschiedene Jahrgänge; Berechnungen der Autorinnen.

Zusammenfassend zeigt sich also: In den drei näher analysierten Erwerbsverlaufstypen, in denen Minijobs eine Rolle spielen, arbeiten die jüngsten Frauen sowie die am schlechtesten ausgebildeten Frauen überdurchschnittlich lange in Minijobs. Insgesamt zeigt sich ein klarer Ost-WestUnterschied. Während stabile Teilzeitverläufe zu größeren Anteilen bei Frauen in Westdeutschland anzutreffen sind, kommen die diskontinuierlich-prekären Verläufe zu größeren Anteilen bei ostdeutschen Frauen vor. Betrachtet man alle Frauen mit Kindern, dann weisen ihre Erwerbsbiografien überwiegend Teilzeiterwerbsverläufe auf, während sie gleichzeitig mit Partner im Haushalt leben. Mit diesem Erwerbsverlauf kann - ob gewollt oder unfreiwillig - die eigene Existenz nicht gesichert und dem neuen Leitbild des Adult-Worker-Model nicht entsprochen werden.

Wir können also anhand der Erwerbsverlaufstypen die Frauengruppe identifizieren, die das Bild der Minijobberin in der Öffentlichkeit bestimmt: Durch den Partner abgesichert versorgen sie die Kinder und erwerben mit dem Minijob einen Hinzuverdienst, und da sie gering qualifiziert sind, haben sie auch nicht viele Alternativen. Dass diese Gruppe vergleichsweise am längsten in dieser nicht existenzsichernden Erwerbsform verbleibt, deutet auf eine relativ stabile Situation hin, wie sie auch in Betriebsuntersuchungen gefunden wurde (Voss-Dahm 2009, S. 175ff.).

Wir finden aber ebenfalls - und das ist das bemerkenswerte Ergebnis -, dass Minijobs auch im Rahmen diskontinuierlicher Verläufe vorkommen, bei Frauen ohne Partner, bei Frauen, die keine Kinder versorgen, als Bestandteil eines Verlaufes mit vielen Wechseln, die auch beruflich gut ausgebildete Frauen absolvieren. Inwieweit diese häufigen Wechsel des Erwerbsstatus und auch die Minijobs erwünscht sind, wissen wir bisher nicht. Auch über die Haus-
ABB. 2

\section{Pfadabhängigkeit von Erwerbsverläufen - Vorerfahrung in Arbeitslosigkeit -}

durchschnittliche Anzahl Jahre vor 2001

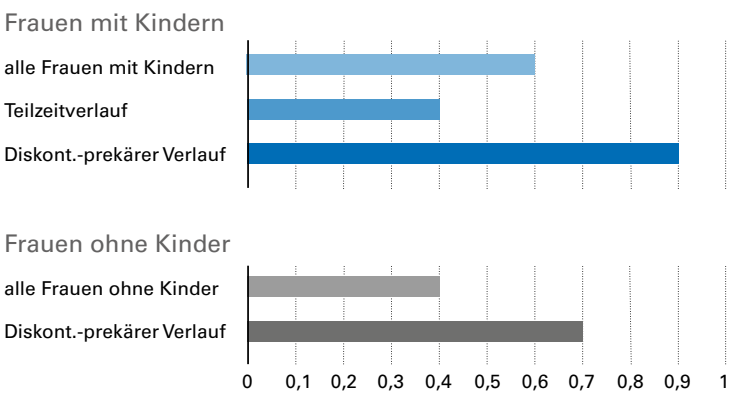

Frauen mit Kindern

lle Frauen mit Kindern

Teilzeitverlau

Quelle: SOEP, verschiedene Jahrgänge; Berechnungen der Autorinnen.

haltseinkommen sowie die Arbeitszeitwünsche der Frauen - differenziert nach deren Erwerbsverlaufstypen - liegen bislang nur wenige Erkenntnisse vor. Wir analysieren im Folgenden die Einkommensposition der Haushalte, in denen Minijobberinnen leben, anhand der Haushaltsnettoäquivalenzeinkommen ${ }^{\oplus}$ (4.2) sowie die Frage, ob ein Beschäftigungsverhältnis zu Minijob-Konditionen freiwillig oder unfreiwillig eingegangen wird (4.3).

\subsection{Einkommen in den Erwerbsverlaufstypen mit Minijob}

Am Ende des Beobachtungszeitraumes im Jahre 2007 zeigt sich vor allem im diskontinuierlich-prekären Verlauf (MK-2) der Frauen mit Kindern ein klares Ungleichgewicht: Ein weit überdurchschnittlicher Anteil dieser Haushalte (42\%) ist im untersten Quartil der Einkommensverteilung zu finden (Tabelle 2). Diese Haushalte haben ein weit unterdurchschnittliches Median-Haushaltsäquivalenzeinkommen von nur 1.212,10€ zur Verfügung. Damit

(1) Die Berechnungen wurden auf Basis der neuen bzw. modifizierten OECD-Skala durchgeführt. Zur Ermittlung des Äquivalenzeinkommens wird die Summe der Nettoeinkommen der Haushaltsmitglieder gebildet und durch die gewichtete Anzahl der Haushaltsmitglieder dividiert. Die Person mit dem Haupteinkommen hat den Faktor 1,0. Alle anderen Mitglieder der Bedarfsgemeinschaft im Alter von 14 Jahren und älter werden mit dem Faktor 0,5 und alle Jüngeren mit 0,3 gewichtet. Damit wird mit den Haushaltsäquivalenzeinkommen das Alter und die Anzahl der damit zu versorgenden Personen mitberücksichtigt. 
wird vor allem in Haushalten, in denen Frauen dieses Verlaufs leben, das Minijobeinkommen der Frau dringend benötigt. Umgekehrt kann aber das Minijobeinkommen der Frau den Haushalt nicht aus einer armutsnahen Lage herausführen.

Obwohl die Haushaltsäquivalenzeinkommen in den anderen beiden Verlaufstypen etwas stärker streuen, liegen sie auch hier überdurchschnittlich häufig im untersten Quartil (Tabelle 2).Vor allem für den stabilen Teilzeiterwerbsverlauf gilt aber auch, dass über die Hälfte $(53,2 \%)$ der Frauen in diesem Verlaufstyp Tätigkeiten in Teilzeit oder Minijob tatsächlich als Hinzuverdienst zu einem höheren Einkommen ihrer Lebenspartner in Haushalten der beiden oberen Einkommensquartile leistet.

Es bestehen bei allen Verlaufstypen positiv signifikante Zusammenhänge zwischen den Haushaltseinkommen in den Jahren 2001 und 2007..$^{\circledR}$ Dies weist auf eine Pfadabhängigkeit des Haushaltseinkommens innerhalb der Verlaufstypen hin. Das Ausmaß der Pfadabhängigkeit lässt sich an den jeweiligen finanziellen Veränderungen im Haushaltsäquivalenzeinkommen zwischen 2001 und 2007 erkennen. In den drei Verlaufstypen steigen die Medianeinkommen sehr unterschiedlich an (Tabelle 2). ${ }^{\circledR}$ In Haushalten mit Frauen im diskontinuierlich-prekären Verlauf (OK-2) stagnieren sie faktisch, während sich die bereits gute Einkommensposition der Haushalte mit Frauen der stabilen Teilzeiterwerbsverläufe (MK-1) über die Jahre hinweg noch mehr verstärkt. Dagegen festigen sich die sowieso schon schwachen finanziellen Positionen der prekären Verläufe durch nur einen minimalen finanziellen Zuwachs ${ }^{\circledR}$ im Haushaltseinkommen.
Zusammengefasst scheint es hauptsächlich für die Frauen des Teilzeiterwerbsverlaufs (MK-1) zuzutreffen, dass die Paare sich vor dem Hintergrund der gegebenen Anreize und Vereinbarkeitsbedingungen im Haushaltskontext rational dafür entscheiden, die Rollen zwischen Mann und Frau so aufzuteilen, dass die Frau in Teilzeitarbeit und mit Minijob einen Hinzuverdienst erwirbt. Bei den anderen, prekären Verläufen scheint dieses Erwerbsmuster eher aufgrund finanzieller Notwendigkeiten und fehlender Alternativen gewählt oder erzwungen zu sein und führt zunehmend in schlechtere Einkommenspositionen im Haushalt.

Schlechter ausgebildete jüngere Frauen in Haushalten in unterer Einkommenslage sind zu einem Teil gezwungen, diskontinuierlich-prekäre Erwerbsverläufe zu akzeptieren. Sie sind keineswegs in jedem Fall und durchgehend durch einen Partner abgesichert. Erst recht nicht trifft die Annahme zu, dass Minijobberinnen immer ihr Einkommen zu einem hohen bis mittleren Einkommen des Lebenspartners hinzuverdienen. Unter den Frauen in den diskontinuierlichprekären Verlaufstypen (MK-2 und OK-2) sind auch Arbeitnehmerinnen, die mangels Alternative mit Minijobs vorliebnehmen müssen. Diese Frauen sind keineswegs

(1) Die Pearsons Korrelationskoeffizienten betragen für MK-1: 0.45, für MK-2:0.30 und für OK-2: 0.42 .

(B) Allerdings wurden die Daten nicht inflationsbereinigt.

(19) Wenn dieser sehr geringe Zuwachs inflationsbereinigt wäre, wäre sogar eine Einkommensminderung wahrscheinlich.

TABELLE 2

Haushaltsnettoäquivalenzeinkommen 2007 nach Verlaufstypen

\begin{tabular}{|c|c|c|c|c|}
\hline \multirow[b]{3}{*}{ Verlaufstyp } & \multicolumn{3}{|c|}{ Frauen leben überwiegend ... } & \multirow{3}{*}{\begin{tabular}{|l} 
Alle Frauen \\
\\
Anteil Kohorte an \\
Verlaufstyp (in \%)
\end{tabular}} \\
\hline & \multicolumn{2}{|c|}{ mit Kind(ern) } & \multirow{2}{*}{$\begin{array}{c}\text { ohne Kind(er) } \\
\begin{array}{c}\text { Diskont.-prekärer } \\
\text { Verlauf (OK-2) }\end{array}\end{array}$} & \\
\hline & $\begin{array}{l}\text { Teilzeit-Verlauf } \\
\text { (MK-1) }\end{array}$ & $\begin{array}{l}\text { Diskont.-prekärer } \\
\text { Verlauf (MK-2) }\end{array}$ & & \\
\hline $\begin{array}{l}\text { Anteile Haushaltsnettoäquivalenz- } \\
\text { einkommen } 2007 \text { in Quartilen }\end{array}$ & Anteil in \% ( $\mathrm{N}=396)$ & Anteil in \% ( $\mathrm{N}=245)$ & Anteil in \% ( $\mathrm{N}=186)$ & Anteil in \% ( $N=1598)$ \\
\hline unterstes Quartil & 27,6 & 42,3 & 29,2 & 25,0 \\
\hline 2. Quartil & 19,2 & 28,9 & 32,4 & 25,0 \\
\hline 3. Quartil & 30,7 & 21,9 & 18,4 & 26,5 \\
\hline Oberstes Quartil & 22,5 & $(6,9)$ & 20,0 & 23,5 \\
\hline Gesamt & 100 & 100 & 100 & 100 \\
\hline $\begin{array}{l}\text { Median Haushaltsnettoäquivalenz- } \\
\text { Einkommen im jeweiligen } \\
\text { Verlaufstyp } 2007 \text { in } €\end{array}$ & $1.616,00 €$ & $1.212,10 €$ & $1.402,70 €$ & $1.553,00 €$ \\
\hline $\begin{array}{l}\text { Veränderung Median Haushalts- } \\
\text { nettoäquivalenz-Einkommen von } \\
2001 \text { bis } 2007 \text { in Prozentpunkten }\end{array}$ & $+26,3$ & $+2,1$ & $+0,4$ & $+17,7$ \\
\hline \multicolumn{4}{|l|}{ () Fallzahl < 30 . } & WSI Mitteilungen \\
\hline
\end{tabular}


materiell gut abgesicherte Ehefrauen, die im Rahmen einer Wahlfreiheit des Lebensmodells einen Minijob wählen.

\subsection{Arbeitszeiten in den Erwerbsverlaufstypen mit Minijobs}

Inwiefern die Entscheidung für eine Teilzeit- oder Minijobtätigkeit tatsächlich freiwillig getroffen wurde, kann anhand des Vergleiches von tatsächlichen und gewünschten Arbeitszeiten in den einzelnen Verlaufstypen betrachtet werden.

Unsere Analysen im Längsschnitt zeigen, dass die in Minijobs tätigen Frauen des Teilzeiterwerbsverlaufs am häufigsten und am längsten mit ihren Arbeitszeiten zufrieden sind: Weit überdurchschnittlich arbeitet über ein Viertel der Frauen des Teilzeiterwerbsverlaufs (MK-1) längere Zeit (drei bis vier Jahre) übereinstimmend mit ihren Arbeitszeitwünschen (ohne Tabelle). ${ }^{20}$ Bei ihnen stimmen im Mittel gewünschte und tatsächliche Arbeitszeiten am längsten überein. Das heißt, sie arbeiten über längere Zeiträume freiwillig in Minijobs - ein Befund, der aber vor dem Hintergrund der gegebenen Anreize und der schwierigen Bedingungen der Vereinbarkeit von Familie und Beruf gesehen werden muss. Aus der Analyse muss festgehalten werden, dass Frauen sich angesichts der Umstände und (Fehl)Anreize zu einem Teil in diesen nicht existenzsichernden Jobs eingerichtet haben. Die riskanten Aspekte im Lebensverlauf nehmen sie in Kauf - oder müssen sie mangels Alternativen akzeptieren. Es können daher keine Aussagen darüber getroffen werden, wie diese Frauen und ihre Partner entscheiden würden, wenn andere Anreizstrukturen und familienfreundlichere Bedingungen gegeben wären.

Bemerkenswert ist, dass es auch in diesem Verlaufstyp Frauen gibt, die gerne längere Arbeitszeiten verwirklichen würden. Auch Wanger (2011) zeigte mit Daten des SOEP für das Jahr 2009, dass insbesondere geringfügig- und teilzeiterwerbstätige Frauen ihre Arbeitszeiten gerne ausweiten würden.

Viel deutlicher als bei Frauen des stabilen Teilzeitverlaufs zeigen sich Diskrepanzen zwischen gewünschten und tatsächlichen Arbeitszeiten bei Frauen in den diskontinuierlich-prekären Verläufen (MK-2, OK-1). Diese Frauen sind unterdurchschnittlich, in geringerem Umfang und über kürzere Zeiträume mit ihren sehr kurzen Arbeitszeiten zufrieden. Vor allem die Frauen ohne Kinder (OK-2) würden gern zu höheren Anteilen und über überdurchschnittlich lange Zeiten hinweg längere Arbeitszeiten verwirklichen. Das heißt, besonders sie sind unfreiwillig in den Minijobs tätig.

\section{Fazit}

Minijobs im Erwerbsverlauf von Frauen haben nicht für alle Frauen die gleiche Funktion und Bedeutung im Lebensverlauf. Die Annahme, dass sie im Rahmen von Teilzeiter- werbsverläufen eher eine Ergänzung zu einem ausreichenden Partnereinkommen darstellen und freiwillig ausgeübt werden, trifft nur für einen Teil der Frauen, nämlich auf etwa $40 \%$ der Frauen mit Kindern zu (Übersicht 1). Sie haben einen stabilen Teilzeiterwerbsverlauf, für den sich die verbreitete Annahme zu bestätigen scheint, dass Minijobs einen willkommenen Hinzuverdienst zu einem höheren Partnereinkommen darstellen. Doch auch hier müssen die Rahmenbedingungen berücksichtigt werden: unzureichende Kinderbetreuungseinrichtungen, ungünstige Arbeitszeitlagen und Wegezeiten tragen ebenso dazu bei, dass Frauen auf Minijobs ausweichen müssen, wie tradierte Rollenvorstellungen. Ansprüche an Mütter, ihren Kinder am Nachmittag für Hausaufgabenhilfe und als „logistische Unterstützung “ für Fahrten zu Freunden und Freizeitaktivitäten zur Verfügung zu stehen, und schließlich damit Hand in Hand einhergehende Leitbilder, nach denen Vollzeitarbeit oder substanzielle Teilzeitarbeit von Müttern nicht wünschenswert sei und schließlich eine geschlechtsspezifische Arbeitsteilung, die männliche Beteiligung an der Haus- und Betreuungsarbeit nicht vorsieht, tragen zu Minijobbeschäftigung von Müttern bei.

Für einen Teil der Frauen des stabilen Teilzeitverlaufs gilt, dass sie den Minijob ausüben, ohne dass Fürsorgepflichten für kleine Kinder zu leisten sind, weil die Paare haushaltsökonomisch rational die gegebenen Anreizstrukturen nutzen. Aus der Perspektive des „Adult-Worker-Model“ wirken diese Anreize als riskante „Fehlanreize“, da sie mit kurzfristigen Vorteilen Frauen dazu verleiten, sich auf dieses Modell einzulassen, das sie auf einen Pfad bringt, der im Lebensverlauf zu Armut führen kann. Volkswirtschaftliche Potenziale einer umfangreicheren Erwerbstätigkeit werden durch solche „Fehlanreize“ ebenso verschenkt (vgl. Voss/Weinkopf in diesem Heft) wie die Chancen der Frauen auf eine eigenständige soziale Sicherung. Wenn das traditionelle Geschlechterrollenmodell heute nicht mehr adäquat ist, da es der eigenständigen Existenzsicherung aller Menschen entgegensteht, müssten konsequenterweise auch die gesetzlichen Regelungen, die eine Begünstigung des tradierten Modells beinhalten, korrigiert werden.

Daneben gibt es eine andere Gruppe von Frauen, deren diskontinuierlich-prekäre und in dieser Form nicht freiwillig gewählte Erwerbsverläufe systematisch durch Minijobs strukturiert sind und die langfristig negative Wirkungen zeigen. Dies trifft für knapp $30 \%$ der Frauen mit Kindern und etwa ein Viertel der Frauen ohne Kinder zu. Diese Verläufe sind besonders häufig in Ostdeutschland anzutreffen. Es handelt sich um diskontinuierlich-prekäre Verläufe von eher schlechter ausgebildeten Frauen, die Minijobs mangels besserer Alternativen ausüben und die finanziell zum Teil in prekären Lagen leben. Ein erheblicher Teil von ihnen hat keinen Partner, auf dessen Einkommen sie bauen könnten.

(20) DieTabelle kann bei den Autorinnen angefordert werden. 
Auch dies ist durch rechtliche Regelungen mit bedingt. Der strikte Vorrang einer Arbeitsaufnahme vor einem Leistungsbezug nach SGB II führt offenbar auch dazu, dass Frauen in Minijobs auch dann arbeiten, wenn kein Partner ihren Lebensunterhalt (ausreichend gut) absichert. Für diese Gruppe von Frauen ist der Minijob nicht erst in der Langfristperspektive riskant, sondern er ist bereits aktuell Bestandteil eines diskontinuierlich-prekären Verlaufs, der in einem Teil der Fälle durch Leistungen des Arbeitslosengeldes (ALG) II aufgestockt werden muss.

Für alle Verläufe mit Minijobs gibt es Hinweise für statusbezogene wie auch finanzielle Abhängigkeiten der weiteren Erwerbsverläufe von vorherigen Status. Damit zeigen sich Minijobs als doppelt riskant beim normativen Übergang zum Adult-Worker-Modell.

\section{LITERATUR}

Aisenbrey, S./Fasang, A. (2007): Beyond Optimal Matching: The "Second Wave" of Sequence Analysis, CIQLE - The center for Research on Inequalities and the Life Course, Yale University, Working Paper 2007-02, New Haven Allmendinger, J. (2009): Frauen auf dem Sprung. Wie junge Frauen heute leben wollen. Die Brigitte-Studie, München

Blossfeld, H.-P./Huinink, J. (2002): Lebensverlaufsforschung als sozialwissenschaftliche Forschungsperspektive:Themen, Konzepte, Methoden und Probleme, Globalife working papers series (28), Bielefeld

Brüderl, J./Scherer, S. (2004): Methoden zur Analyse von Sequenzdaten, in: Diekmann, A. (Hrsg.): Methoden der Sozialforschung, Kölner Zeitschrift für Soziologie und Sozialpsychologie (KZfSS) (Sonderheft 44), S. 330-347

Brzinksy-Fay, C./Kohler, U./Luniak, M. (2006): Sequence analysis with Stata in: The Stata Journal 6 (4), S. 435-460 (letzter Zugriff:11.6.2008)

Eichhorst, W./Marx, P./Thode, E. (2010): Atypische Beschäftigung und Niedriglohnarbeit Benchmarking Deutschland: Befristete und geringfügigeTätigkeiten, Zeitarbeit und Niedriglohnbeschäftigung, Bertelsmann Stiftung, Gütersloh

Europäische Union (EU) (2011): Strategy for Equality between women and men 2010-2015, http://ec.europa.eu/social/main.jsp?catld=418\&langld=de (Zugriff: 30.6.2011)

Fouarge, D./Muffels, R. (2009): Working Part-Time in the British, German and Dutch Labour Market. Scarring for the Wage Career?, in: Schmollers Jahrbuch 129, S. 217-226

Hacket, A. (2012): Erwerbsverläufe in der Haupterwerbsphase - Pluralisierung und Prekarisierung der Erwerbsverläufe?, in: Forschungsverbund Sozioökono mische Berichterstattung (Hrsg.): Berichterstattung zur sozioökonomischen Entwicklung in Deutschland, Wiesbaden, S. 507-532

Hans-Böckler-Stiftung (HBS-Datenbank) (2011): Datenbank atypische Beschäftigung der Hans-Böckler-Stiftung, http://www.boeckler.de/datyp/index php?page=1\&region=D\&sex=m und http://www.boeckler.de/datyp/index. php?page=1\&region=D\&sex=f (letzter Zugriff 06.7.2011)

Kassner, K. (unter Mitarbeit von I. Quander und T. Wandrei) (2011): Zur Vereinbarkeitssituation von Eltern mit Schulkindern, Bundesministerium für Familie, Senioren, Frauen und Jugend (BMFSFJ), Monitor Familienforschung (25), Berlin Klammer, U./Tillmann, K. (2001): Flexicurity: Soziale Sicherung und Flexibilisierung der Arbeits- und Lebensverhältnisse, Forschungsprojekt im Auftrag des Ministeriums für Arbeit und Soziales, Qualifikation und Technologie des Landes NRW, Düsseldorf

Klammer, U./Wilthagen, T./Chung, H./Thiel, A. (2008): Flexibility and Security over the Life Course. Take it or leave it: flexible working time arrangements and the synchronization of business cycle and life cycle, European Foundation for the Improvement of Living and Working conditions, Working paper (2), Dublin

Klenner, C./Klammer, U. (2009): Weibliche Familienernährerinnen in West und Ostdeutschland - Wunschmodell oder neue Prekarität?, in: Bundesministerium für Familie, Senioren, Frauen und Jugend (BMFSFJ) (Hrsg.): Rollenleitbilder und -realitäten in Europa. Rechtliche, ökonomische und kulturelle Dimensionen, Dokumentation des Workshops 20.-22. Oktober 2008, Baden-Baden, S. 62-84
Klenner, C./Schmidt, T. (2011): Teilzeitarbeit im Lebensverlauf von abhängig beschäftigten Frauen, in: Klammer, U./Motz, M. (Hrsg.): Neue Wege - Gleiche Chancen: Expertisen zum Ersten Gleichstellungsbericht der Bundesregierung, Wiesbaden, S. 253-312

Lewis, J. (2004): Auf dem Weg zur "Zwei-Erwerbstätigen"-Familie, in: Leitner, S./Ostner, I./Schratzenstaller, M. (Hrsg.): Wohlfahrtsstaat und Geschlechterverhältnis im Umbruch. Was kommt nach dem Ernährermodell?, in: Jahrbuch für Europa- und Nordamerika-Studien 7, Wiesbaden, S. 62-84

Sachverständigenkommission (2011): Neue Wege - Gleiche Chancen. Gleichstellung von Frauen und Männern im Lebensverlauf. Gutachten der Sachverständigenkommission an das BMFSFJ für den 1. Gleichstellungsbericht der Bundesregierung, Berlin

Schäfer, A./Vogel, C. (2005): Teilzeitbeschäftigung als Arbeitsmarktchance, in: DIW Wochenbericht 72 (7), S.131-138

Schmidt, T./Schmitt, C. (2005): Erwerbsverläufe, in: SOFI/IAB/ISF/INIFES (Hrsg.): Berichterstattung zur sozioökonomischen Entwicklung in Deutschland Arbeit und Lebensweisen, Wiesbaden

Sozio-oekonomisches Panel (SOEP) (2009): Daten für die Jahre 1984-2008, Version 25, Berlin

Statistisches Bundesamt (2006): Datenreport 2006. Zahlen und Fakten über die Bundesrepublik Deutschland, Bundeszentrale für Politische Bildung, Schriftenreihe 544, Bonn

Statistisches Bundesamt (2010), Sozialberichterstattung - Datenbasis: Mikrozensus, http://www.sozialpolitik-aktuell.de/tl_files/sozialpolitik-aktuell/_Politikfelder/Einkommen-Armut/Datensammlung/PDF-Dateien/abblll72.pdf (letzter Zugriff: 01.9.2011

Statistisches Bundesamt (2011): http://www.sozialpolitik-aktuell.de/index. php/familie-datensammlung.html\#viii-tageseinrichtungen-fuer-kinder (letzter Zugriff: 01.09.2011)

Steffen, J. (2010): Gebrochene Erwerbsbiografien, atypische Beschäftigung und drohende Altersarmut, in: Arbeitnehmerkammer Bremen (Hrsg.): Pers pektiven solidarischer Sicherungskonzepte (12), S. 1-11

Tschersich, N./Seifert, H./Herzog-Stein, A./Gensicke, M. (2010): Einmal atypisch, immer atypisch beschäftigt? Mobilitätsprozesse atypischer und normaler Arbeitsverhältnisse im Vergleich, in: WSI-Mitteilungen 63 (4), S. 179-187 Voss-Dahm, D. (2009): Über die Stabilität sozialer Ungleichheit im Betrieb. Verkaufsarbeit im Einzelhandel, Berlin

Wanger, S. (2011): Viele Frauen würden gerne länger arbeiten, IAB-Kurzbericht (9), Nürnberg

Wolf, E. (2010): Lohndifferenziale zwischen Vollzeit- und Teilzeitbeschäftigten in Ost- und Westdeutschland, WSI-Diskussionspapier (174), Düsseldorf

\section{AUTORINNEN}

CHRISTINA KLENNER, Dr., ist Wissenschaftlerin im Wirtschafts- und Sozialwissenschaftlichen Institut (WSI) in der Hans Böckler Stiftung. Arbeitsschwerpunkte: Geschlechterverhältnisse, Frauenerwerbstätigkeit und Work-Life-Balance, Gleichstellung.

\section{christina-klenner@boeckler.de}

TANJA SCHMIDT, Dipl. Soziologin, ist freiberufliche Sozialforscherin in Berlin und Wissenschaftliche Mitarbeiterin an der HSU Hamburg. Arbeitsschwerpunkte: Lebens- und Erwerbsverläufe von Frauen und Männern, Soziale Ungleichheit, Capabilities im Lebensverlauf.

kontakt@schmidt-sozialforschung.de; tanja.schmidt@hsu-hh.de 